\title{
Recent Advances in Acquisition/Reconstruction Algorithms for Undersampled Magnetic Resonance Imaging.
}

\author{
Giuseppe Placidi \\ $A^{2}$ VI-Lab, c/o Dept. of Life, Health, and Environmental Sciences, University of L'Aquila, Via Vetoio Coppito \\ L'Aquila, ITALY \\ giuseppe.placidi@univaq.it
}

\begin{abstract}
Several applications of Magnetic Resonance Imaging (MRI), in particular dynamic MRI and functional MRI (fMRI), require rapid acquisition to measure dynamic processes changes. Experimental data are collected in the k-space by following different trajectories to cover the whole space. Complete data acquisition necessitates waiting for a fixed time interval: a reduced number of collected trajectories allows acquisition time reduction but undersampling occurs, often producing artefacts. In what follows, a review of methods for sparse sampling acquisition and reconstruction is presented.

In particular, a differentiation is done between sparse acquisition methods which do not use any restoration algorithm (artefacts are tolerated) and those methods for which a restoration algorithm is essential. The first class contains also methods where spatial information is shared between temporal images to reduce the collected data. In the second class of methods, a differentiation is done between those reconstruction/restoration methods that reduce artefacts independently of the sample shape, and those restoration methods that adapt their action by modifying the acquisition trajectories during the acquisition, i.e. the chosen trajectories (both in number and directions) are dependent on the sample shape. A third emerging class of methods, those including hybrid forms of the second class, are also reported.
\end{abstract}

\section{Introduction}

In conventional MRI, the number of collected data is determined by spatial resolution requirements and by the Nyquist criterion for the alias-free field of view (FOV) to obtain an image by fully-sampled k-space set. Recently, MRI has developed considerably into the direction of dynamic imaging opening up several new applications such as monitoring of contrast agent dynamics [[1]-[4]], mapping of human brain neural activity based on blood oxygenation level-dependent (BOLD) imaging contrast [[4]], MR-guidance of biopsies [[6]-[8]], monitoring of ablations [[9]-[11]], guidance of intravascular procedures [[12]-15], and real-time visualization of cardiac motion $[16,17]$. Although these developments are generally promising their application is limited by the compromise between temporal and spatial resolution. To improve temporal resolution many approaches use "undersampling". 
The term undersampling indicates that the Nyquist criterion is not satisfied, at least in some regions of $k$-space, i.e. images are reconstructed by using a number of k-space samples lower than that theoretically required to obtain a fully-sampled image.

Undersampling is used for several reasons, such as reducing acquisition time [17-21] and motion artifacts [22], achieving higher resolution [23-25], and balancing the trade-off between spatial and temporal resolution $[26,27]$. Normally undersampling implies image artifacts, often in the form of aliasing or streak structures. Parallel imaging methods such as sensitivity encoding (SENSE) [28], simultaneous acquisition with spatial harmonics (SMASH) [29] and generalized auto calibrating partially parallel acquisition (GRAPPA) [30] also can be thought of as undersampling methods in which artifacts are removed by using information obtained from multiple RF coils and receivers. But, at the end of the process, a complete image is reconstructed through the partial information collected by different receivers.

Time required to fully sample 3D Cartesian $k$-space is relatively long. Alternative non-Cartesian trajectories can provide faster $k$-space coverage and more efficient gradients usage. When very fast volume coverage is required, undersampling strategies can be combined with non-Cartesian trajectories for further reduction of the scan time.

Undersampling can influence the resulting image in different ways depending on the k-space covering paths: Cartesian, radial, spiral, 2D, 3D, etc. (see Figures 1-3).

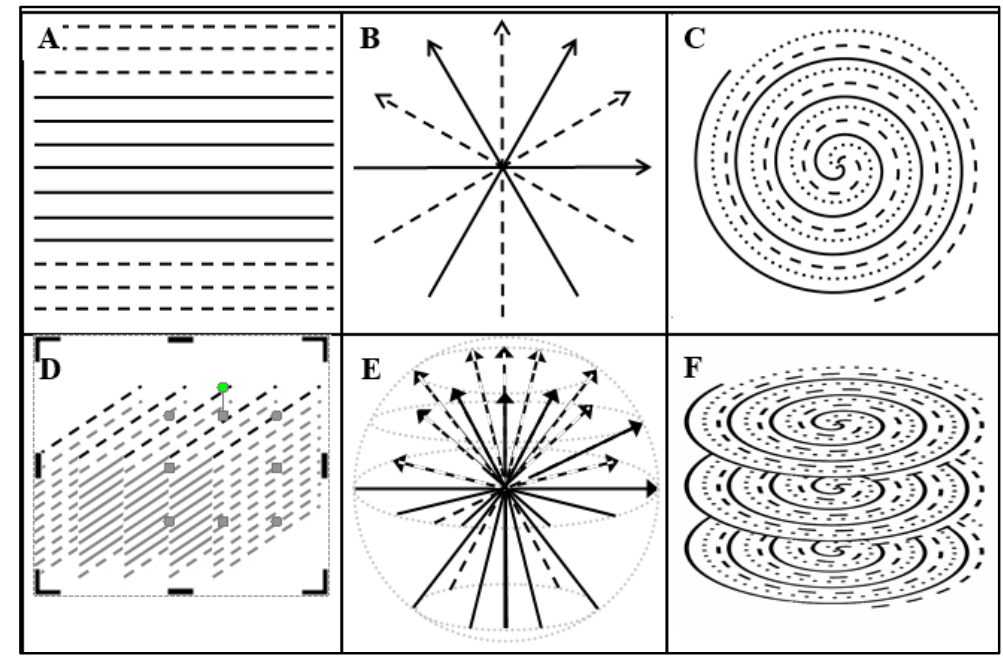

Figure 1. Sparse sampling in 2D (A-C) and 3D (D-F) settings: Cartesian (A, D), Radial (B, E) and Spiral (C, F) samplings. Measured trajectories are represented by continuous lines, missing trajectories are represented by dashed/dotted lines.

This implies that it can occur differently in different k-space regions. For example, when Cartesian sampling is used, undersampling occurs because some rows in the k-space rectangular grid are missing along the phase encoding direction (see Figures $1 \mathrm{~A}$ and $1 \mathrm{D}$ ); in case of radial acquisition, i.e. acquisition from projections, undersampling occurs because some radial directions in the k-space are missing (see Figures $1 \mathrm{~B}$ and $1 \mathrm{E}$ ). As will be clarified later, each of these cases requires a specific restoration algorithm, but all the methods afford the same problem: to reconstruct an image, suppose a 2D image 
Giuseppe Placidi; Recent Advances in Acquisition/Reconstruction Algorithms for Undersampled Magnetic Resonance Imaging, Journal of Biomedical Engineering and Medical Imaging, Volume 1, No 5, Oct (2014) , pp 53-70

$\hat{f}\left(t_{1}, t_{2}\right)$, starting from some collected sparse samples of its Fourier coefficients, $\left.\hat{f}\right|_{\Omega}$ while violating the Nyquist rate. In this paper, a review of the most effective sparse sampling and restoration strategies for solving the previous problem are reported. In particular, a differentiation is done between sparse acquisition methods which do not use any restoration algorithm (artifacts are tolerated) and the sparse acquisition methods for which a restoration algorithm is essential (this differentiation is defined by a temporal transition). The first class of methods contains also those methods that use temporal correlation between dynamic images (when temporal resolution is required) as a sort of restoration algorithm: some k-space coefficients are collected just once and shared by the whole set of dynamic images. The decision to include these methods among those of the first class is that no mathematical strategy is used to estimate the missing coefficients: the missing k-space coefficients are supplied from a reference image to the whole set of dynamic images. In the last class of methods, a differentiation is done between those that adapt their strategy to a given acquisition method, trying to reduce artifacts independently of the sample shape, and those that start their action by modifying the acquisition trajectories during acquisition, i.e. the acquisition trajectories (both in number and in directions) depend on the sample shape. Finally, hybrid methods, that are emerging by those allowing to the second class, are presented.

\section{Sparse Methods without Restoration}

The method proposed by Liao et al. [22] uses a variable-density stack of spiral trajectories which varies the sampling density both along the $k x-k y$ plane and the $k z$ direction. The method is shown to preserve reasonable image quality while reducing the acquisition time by approximately half compared to a fullysampled stack of spirals. Vastly undersampled 3D projections are used to increase temporal resolution and provide better dynamic information for 3D contrast-enhanced angiography (CE)-MRA. Aliasing caused by undersampling in this method often can be tolerated because the vessel-tissue contrast is high. In [32] variable density k-space sampling trajectories are used to restrict the violation of the Nyquist criterion to the outer part of the k-space. Spiniak et al. [20] use an efficient undersampling method for a progressive missile guidance trajectory. The purpose of this work is to examine $k$-space undersampling in conjunction with the shells trajectory. The shells method is a non-Cartesian 3D $k$-space trajectory [33]: fully sampled concentric spherical shells are used for 3D selective RF pulse design [34]. The same sampling strategy is used for a single $k$-space shell acquisition that acts as a spherical navigator echo for motion tracking [35]. A full 3D $k$-space shells acquisition is implemented in [36] and it is demonstrated to have motion correction properties in [37].

Because the shells trajectory does not acquire data on the "corners" of $k$-space, it offers a volume coverage reduction compared to a fully-sampled 3D Cartesian acquisition. This is substantially larger than the $k$-space volume coverage reduction obtained by skipping views in the phase encoded corners of a Cartesian 3D acquisition. The shells trajectory samples $k$-space on the surfaces of a series of concentric spheres. In reference [38] interleaved helical spirals sweep on the surface of each shell from one pole to another (see Figure 2). 


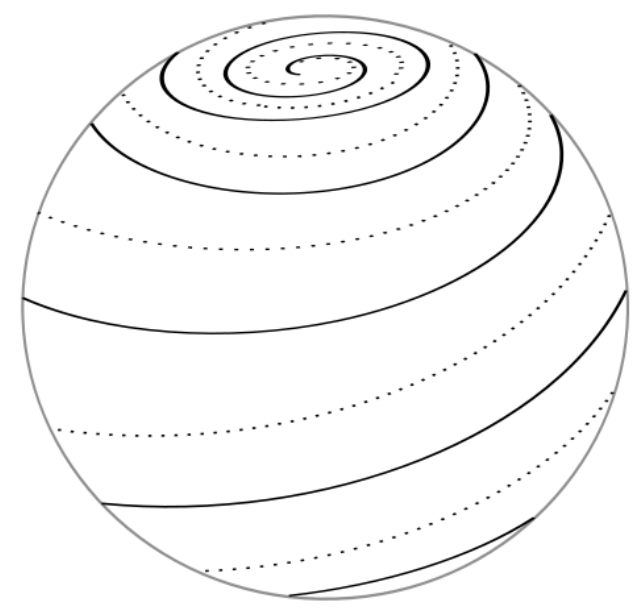

Figure 2. Shells $k$-space trajectories. Spiral interleaves, coded by continuous and dotted lines respectively, sweep from one pole to the other of a spherical surface. In an undersampling scheme, some interleaves are skipped, for example that represented by a dotted line.

The shells acquisition method is a true center-out trajectory in 3D $k$-space, in that it starts from the origin of the $k$-space and extends progressively to the periphery. An important property of the shells trajectory is that a large number of interleaves exclusively collect data from the periphery without crossing the center of $k$-space. Because the number of interleaves varies on a per-shell basis, there is flexibility in controlling the sampling density as a function of the $k$-space radius. These properties suggest that the shells trajectory is well suited for undersampling design. The way used to implement undersampling for the shells trajectory method is to remove selected interleaves within a shell. This method is proven to be an optimal balance between acquisition time reduction, undersampling artifacts reduction and edges preservation.

In PROPELLER (periodically rotated overlapping parallel lines with enhanced reconstruction) [39,40], data acquisitions consist in a multiple-shot fast spin-echo approach in which several parallel k-space lines are collected in each time of repetition (TR), forming a blade that is then rotated around its center and acquisition is repeated to cover the k-space (see Figure 3).
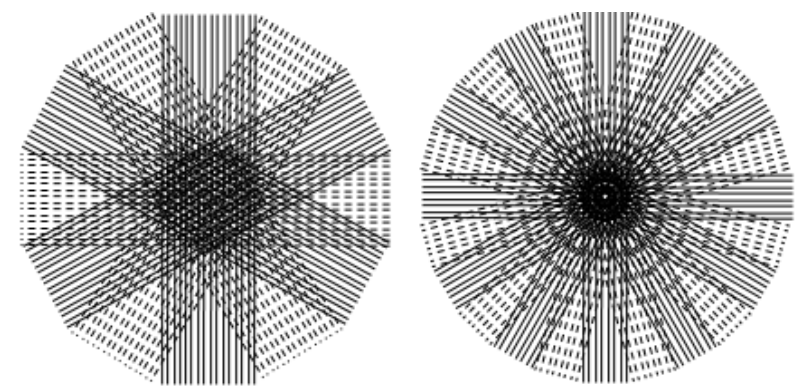

Figure 3. Different PROPELLER k-space sampling patterns. In particular: (A) shows a 6 blades scheme, each blade composed by 16 lines; (B) shows a 12 blades scheme, each blade composed by 8 lines. Undersampling is realized by skipping dotted blades. 
Giuseppe Placidi; Recent Advances in Acquisition/Reconstruction Algorithms for Undersampled Magnetic Resonance Imaging, Journal of Biomedical Engineering and Medical Imaging, Volume 1, No 5, Oct (2014) , pp 53-70

The acquisition is radial. PROPELLER imaging is an MRI data acquisition and reconstruction that is attracting due to its greatly reduced sensitivity to various source of image artifacts (first of all motion artifacts). In ref. [41] PROPELLER is used in undersampled form and the resulting artifacts are studied.

Other methods utilize information redundancy from temporal correlations of dynamic images as a sort of restoration. Keyhole methods [42,43], selective acquisitions [44, 45] and reduced-encoding MR imaging by generalized series reconstruction (RIGR) [46] assume temporal variation is primarily from the central portion of k-space. In the keyhole fMRI technique $[47,48]$, a full k-space reference image is acquired for each cycle and for each activation state. To reduce acquisition time, the strategy for the other images in each cycle consists of sampling only a central fraction of the k-space (low k-space data). The missing peripheral lines in the k-space (high k-space data) are supplied from the reference images. In this way, an approximately $60 \%$ in acquisition time per image can be saved for $\mathrm{T}_{2}{ }^{*}$-weighted gradientecho schemes. The resultant keyhole fMRI images retain nearly all the functional information of the original high spatial resolution images while substantially reducing the acquisition time [48]. However, two potential problems, associated with the keyhole technique, may affect the reconstructed images: noise correlation between different images and discontinuities. Because of data sharing, high k-space data are the same for all the keyhole images. Therefore, noise between images is correlated. When statistical analysis is performed across a series of images to assess significant activations, the shared kspace data will decrease the effective sampling size and result in a higher noise level.

Regarding discontinuities, the basic principle of the keyhole technique is to combine the dynamic low kspace data with the reference high k-space data. Due to the "image to image" signal fluctuation, amplitude and phase discontinuities may exist at the junction of the high k-space and the low k-space data. These discontinuities may result in artifacts and spatial resolution reduction. A detailed description of the effects of the key-hole method can be found in [49].

Clinical practice shows that a high spatial resolution is essential for most dynamic imaging applications. To this purpose, some technique increases temporal and/or spatial resolution by directly exciting the region of interest, such as the zoomed imaging technique [50]. Beyond finding the optimal compromise between temporal resolution, spatial resolution and SNR for a specific application, it is desirable to find ways to introduce some additional information into the imaging process and so to truly enlarge the amount of information available without spending additional measuring time. In this sense, reduced field-of-view (rFOV) reconstruction utilizes the fact that only a limited region of an image undergoes substantial variation over time [51,52]. The idea of rFOV is to exploit the fact that dynamic changes in an image series may be confined to a certain area (rFOV) within the full FOV. This information can be used to increase temporal resolution in fluoroscopic imaging without sacrificing spatial resolution. Having been demonstrated first for Cartesian sequences [[51]] it is applicable to any segmented k-space acquisition scheme as radial [53-55] or spiral imaging [56]. The principal limitation of such a technique is that erroneous results will be produced if some of the additional information is wrong or the required conditions are not filled, e.g., if dynamic changes of the object outside the rFOV do occur. Because in clinical practice it will generally be difficult to strictly satisfy such conditions, it is important to evaluate the respective robustness of the different implementations of the rFOV technique. The projectionreconstruction rFOV approach (PR rFOV) has been demonstrated to be more robust than the other rFOV 
approaches. The greater robustness is a consequence of the special aliasing properties of projectionreconstruction imaging and is considered to be crucial for clinical applications.

Both Keyhole and rFOV methods are based on the fact that the information from relatively stationary regions in object space is considered to be redundant.

The method UNFOLD (UNaliasing by Fourier-encoding the Overlaps using the temporaL Dimension) $[57,58]$ supposes that data have to be collected both in spatial and in temporal directions: the same image has to be reconstructed more times to reproduce temporal variations occurring inside it. UNFOLD uses the $t$ axis of $k-t$ space to resolve information normally encoded along the $k$ axes. In some dynamic applications in which the time axis is not efficiently exploited by conventional encoding, such a reorganization of $\mathrm{k}-\mathrm{t}$ space can lead to a significant decrease in acquisition time for the temporal frames. In this case, spatial aliased versions of the same image (each image having a determined phase shift offset) are collected: spatial aliasing is eliminated by separating overlapped information through the temporal Fourier transform. This can lead to a temporal resolution improvements by nearly a factor of two in cardiac-triggered imaging, and by as much as a factor of eight in fMRI. Image reconstruction process requires aliasing elimination (through temporal Fourier transform) before the spatial Fast Fourier Transform. Depending on the situation, the acquisition time reduction of a temporal frame can be translated into a reduction of the total imaging time, into an improvement of the spatial or temporal resolution, or into an increase in the spatial coverage. The reduction can also be used to allow a faster pulse sequence to be replaced by a slower one while preserving time resolution. This method cannot be used to improve spatial resolution when temporal data are not necessary or not available.

Temporal acceleration can be achieved with the k-t broad-use linear acquisition speed-up technique ( $k-t$ BLAST), which uses training data as prior information along with the interleaved sampling function [59]. Conceptually, UNFOLD and k-t BLAST reduce the information redundancy by economically using k-t space (the combination of k-space with a time axis [60]). Originally, k-t BLAST has been used with Cartesian k-space trajectories $[59,60]$, due to the simplicity of describing and correcting for the aliasing produced by rectilinear sampling patterns. After that, it has been also presented as a non-Cartesian sampling/reconstruction method [61]. However, spiral readouts are appropriate for certain applications [62] because they have good flow properties and are time efficient, which enables further increases in spatial and/or temporal resolution when combined with under sampling techniques. Shin et al. [63] solve elegantly the problem for spiral trajectories. An improvement to the UNFOLD method is proposed in [64] where UNFOLD is combined with the under sampled 3D stack of spirals acquisition [65] to increase spatial coverage for high resolution fMRI.

\section{Sparse Methods with Restoration}

\subsection{Sample Independent Acquisition/Restoration Methods}

A first group includes those methods where the redundancy sources are inherent properties of the image but the image properties (for example sparseness or symmetries) are not used while performing data acquisition.

Placidi et al. [66] describe an algorithm which is effective in reducing truncation artifacts due to missing k-space samples in MRI. The algorithm works first by filling the incomplete matrix of coefficients with 
Giuseppe Placidi; Recent Advances in Acquisition/Reconstruction Algorithms for Undersampled Magnetic Resonance Imaging, Journal of Biomedical Engineering and Medical Imaging, Volume 1, No 5, Oct (2014) , pp 53-70

zeroes and then adjusting, through an iterative process, the missing coefficients by performing a reduction of the undersampling artifacts before the image reconstruction through a Fourier Transform method [67]. Then, this set of coefficients is used as a basis for a superresolution algorithm that estimates the missing coefficients by modeling data as a linear combination of increasing and decreasing exponential functions, through the Prony's method. The Prony's method consists on the interpolation of a given data set with a sum of exponential functions: the MRI signals can be well represented as a sum of exponential functions and the missing data can be extrapolated by this representation. The algorithm performs very well, both for Cartesian missing rows scheme (phase-frequency acquisitions) and for radial-missing angle (acquisition from projections) undersampling, but it requires some computational overhead. A simpler variation of this method is reported in [68] where a simple constraint for iterative reconstruction, capable to deal with any sparse acquisition method, is used. The methodology herein suggested is based on the attempt to fill in the missing complex k-space values iteratively, making the assumption that the image has to be zero outside a compact support. This approach transforms the original problem into an interpolation problem in the complex domain. The novelty is that it deals with iterative interpolation in the k-space based on the elimination of the artifacts from an extended support of the reconstructed image. The results, simulating different sparse acquisition strategies (Cartesian, radial, and spiral sampling), are not significantly different from those obtained from other, more complicated, iterative methods [66]. Residual artifacts are reduced by using the method reported in [69].

During the last few years, the emerging theory of compressive (or compressed) sensing (CS) [70-74] has offered great insight into both when and how a signal may be recovered to high accuracy (or, for some instances, exactly) even when sampled significantly below the Nyquist rate.

CS requires the measurement of a relatively small number of "random" linear combinations of the signal values (much smaller than the number of signal samples nominally defining it). However, because the underlying signal is compressible, the nominal number of signal samples is a gross overestimate of the "effective" number of "degrees of freedom" of the signal. As a result, the signal can be reconstructed with good accuracy from relatively few measurements by a convex constrained optimization procedure. In MRI the sampled linear combinations are simply individual Fourier coefficients (k-space samples) and CS can be used. In that setting, CS is claimed to be able to make accurate reconstructions from a small subset of k-space, rather than an entire k-space grid. The original paper by Candès et al. [70] is dedicated at random undersampling of Fourier coefficients that is the practical situation of MRI. In order to reconstruct a complete image from the undersampled problem, the simpler strategy assumes that the Fourier coefficients at all of the unobserved frequencies are zero (thus reconstructing the image of "minimal energy" under the observation constraints). This method does not perform very well because the reconstructed image, has severe non local artifacts caused by angular undersampling [70]. A good reconstruction algorithm, it seems, would have to guess the values of the missing Fourier coefficients, i.e. to interpolate $\hat{f}\left(\omega_{1}, \omega_{2}\right)$. However, the prediction of Fourier coefficients from their neighbours are very delicate, due to the global and highly oscillatory nature of the Fourier transform. The prediction can be more efficiently done through convex optimization. To recover $f$ from partial Fourier samples, a solution $f^{*}$ is found for the optimization problem 


$$
\min \|g\|_{T V} \text { subject to } \hat{g}(\boldsymbol{\omega})=\hat{f}(\boldsymbol{\omega}) \text { for all } \boldsymbol{\omega} \in \Omega
$$

Where $\|g\|_{T V}$ represents the total-variation norm of a $2 \mathrm{D}$ object $g$ that, for discrete data $g\left(t_{1}, t_{2}\right), 0$ $\leq t_{1}, t_{2} \leq \mathrm{N}-1$, has the following form

$$
\|g\|_{T V}=\sum_{t_{1}, t_{2}} \sqrt{\left|D_{1} g\left(t_{1}, t_{2}\right)\right|^{2}+\left|D_{2} g\left(t_{1}, t_{2}\right)\right|^{2}}
$$

and

$$
D_{1} g=g\left(t_{1}, t_{2}\right)-g\left(t_{1}-1, t_{2}\right), D_{2} g=g\left(t_{1}, t_{2}\right)-g\left(t_{1}, t_{2}-1\right)
$$

As it is, this technique allows just to reduce artifacts with respect to zero filling of the missing Fourier coefficients (with the exception of the first example reported in [70]). To allow an accurate reconstruction, though in presence of a certain undersampling degree, it is necessary to fill three requirements:

Transform sparsity: the desired image should have a sparse representation in a known transform domain (i.e., it must be compressible by a transform coding).

Incoherence of undersampling artifacts: the artifacts in linear reconstruction caused by $k$-space undersampling should be incoherent (noise like) in the sparsifying transform domain.

Nonlinear reconstruction: the image should be reconstructed with a nonlinear method that enforces both sparsity of the image representation and consistency of the reconstruction with the acquired samples.

The first condition ensures the unknown image can be represented with a lower number of coefficients than that required by Nyquist criterion. MR images meet the first condition. The second condition ensures the position of the sampled coefficients is casual, i.e. they are collected without a specific, deterministic, pattern: this allow the undersampling artifacts have to be distributed as uncorrelated noise. Incoherence is important: MR acquisition can be designed to achieve incoherent undersampling. The third condition is ensured by the presented non-linear reconstruction method. More details on this very promising method applied to MRI can be found elsewhere $[73,74]$. Though very promising, CS can be efficiently applied if the undersampling is not too different from the Nyquist's rate. In fact, most CS applications, especially within medical imaging, have centred on the $I_{1}$-minimization problem due to the fact that the corresponding $1_{0}$-minimization problem is untractable. An interesting recent improvement of CS regards the proposal of an innovative Homotopic I0-Minimization [75]. In this paper [75], Trzarsko et al. describe a method for reconstructing MR images at sampling rates even further below that which are achievable using $I_{1}$-based CS methods by directly attacking the ideal $I_{0}$-minimization problem. The $I_{0}$ minimization problem is described along with both its applied and theoretical implications. Moreover, a practical scheme is presented for addressing the $I_{0}$ quasi-norm based on homotopic approximation using a wide class of deformable sparse priors, and an efficient semi-implicit numerical scheme for computation is described. The authors demonstrate both the problem tractability and the goodness of 
Giuseppe Placidi; Recent Advances in Acquisition/Reconstruction Algorithms for Undersampled Magnetic Resonance Imaging, Journal of Biomedical Engineering and Medical Imaging, Volume 1, No 5, Oct (2014) , pp 53-70

their results, when compared to the classical $\mathrm{I}_{1}$-based CS methods, in spite of a reduction of the used samples for reconstruction.

Knopp et al. [76] present interesting results about the iterative reconstruction from non-uniform k-space sampled data though data sparsity is not reflecting CS requirements. In particular, they discuss about the effectiveness of using non-equispaced FFT to reconstruct images directly from radial or spiral directions, through a generalization of the gridding process [77]. Very accurate results are obtained by using an iterative method to estimate density compensation weights, taking the result of gridding as a starting point. The best gridding results are obtained using the more expensive Voronoi weights. However, substantial improvement of the reconstruction quality is achieved during a small number of iterations for all used trajectories and weights. It should be very interesting to use the method they proposed for image reconstruction from severely undersampled data.

Also Block et al [78] afford the problem of severely undersampled radial data in an iterative way with the usage of a total variation (TV) constraint for the final image to reduce the strike artifacts produced by radial undersampling: also in this case the sparse samples do not fill the CS requirements. The reconstruction is obtained as a non-linear optimization problem, solved through the conjugate gradient method. The conjugate gradient method is an iterative two-step scheme, which is repeated until a satisfying solution has been found. First, a search direction is estimated and, second, a line search into that direction is performed until the minimum of the functional in this direction has been identified. The search direction is obtained by calculating the gradient at the actual estimate and by superposing it with the prior search direction scaled by a factor that guarantees the conjugacy of successive search directions. At every step of the algorithm, the actual image estimate is mapped to the frequency domain. It is then compared how well the estimate fits to the measured data by calculating the difference. If the estimate is good enough, then the residuum vector contains only small entries, otherwise it contains large entries. In this case, the algorithm needs to know how to modify the image estimate in order to improve the match of the samples in the frequency domain. This information is obtained by mapping the residuum back to the image space. The reconstruction of an undersampled radial image through optimization still leads to streaking artifacts: the procedure does not measure the accuracy of the estimate at any other position in k-space than at the positions of the measured k-space coefficients. Being an underdetermined problem, more than one solution exist. To overcome this limitation, a penalty function is introduced into the optimization problem, based on the total variation constraint. The basic assumption of this idea is that the object consists of areas with constant (or only mildly varying) intensity, which applies quite well to medical tomographic images. If the object is piecewise constant, then the best representation of all image estimates that match at the spoke positions should be given by the one with the lowest derivatives at all pixel positions, that is the one minimizing the total variation, represented as the summation of the modules of the image second order derivatives (as discussed by the authors, the choice for the best derivative order still remain a debated argumentation). The obtained results are quite good regarding artifacts reduction, but some blurring occurs.

\subsection{Sample Adaptive Acquisition/Restoration Methods}

Different approaches are based on driving the acquisition process in order to adapt the collected signals to the sample shape. As first example of these methods, Contreras et al. [79] propose a sparse sampling 
method for MRI non-destructive analysis of woods logs, taking advantage of the log cylindrical symmetry by acquiring transverse 1-D projections with a helical and undersampled pattern. Linear interpolation is used to estimate the skipped data and slice images are reconstructed by filtered back-projection. The sequence is improved using selective multi-pass scanning, without major variations of the scan time. The technique is particularly useful for tree logs, since they present several characteristics that can be used to reduce the long scan time, but similar techniques can be also studied for MRI in humans.

Placidi et al. [80-82] present an adaptive acquisition technique for MRI from projections, first defined in the image space [80] and then in the k-space $[81,82]$, to reduce the total acquisition time by collecting just the most informative projections, without any a-priori information about the sample, but using information about the sample collected while acquisition proceeds. This is possible through the calculation of a function, called the entropy of a projection, which is able to measure the information content of the projections during the acquisition process, useful to discover sample internal symmetries, smooth or regular shape. In the k-space method [82] the entropy function is defined on the power spectrum of the projections. The process starts by measuring the projections at four regular orientations: $0^{\circ}, 45^{\circ}, 90^{\circ}$, and $135^{\circ}$. Then the evaluation of the information content of these four initial projections is performed, followed by the selection of new angles where the information content is maximum. The entropy of the power spectrum of each of these starting projections is evaluated and the next projection is measured between the two where the entropy function has a maximum. The procedure is repeated until the difference in entropy remained above a given threshold. The method makes it possible to reduce the total acquisition time, with little degradation of the reconstructed image, adapting itself to the arbitrary shape of the sample (being able to catch eventual internal symmetries, low dynamic range and regular shape). The choice of, approximately, the most informative projections is made during the acquisition process, taking into account the information content of the previous projections. The method allows the acquisition of a near optimal set of projections, close to the most informative set of projections composed by the minimum number of projections, but not the optimal one. In fact, though very effective in reducing the acquisition time and undersampling artifacts, this method suffers from the following limitations: some important projections can be excluded from the acquired set, especially in the proximity of entropy function minima or maxima; some redundant projections can be collected, especially in the proximity of entropy function sharp variations. An effective application of the previous adaptive acquisition method has been also presented as a medical image compression strategy [83].

The method described in [84] considers the problem of measuring exactly the most informative set of projections by collecting a-priori information about the sample through the preliminary measurement of two circular paths at different distances from the k-space centre. The idea behind the algorithm is that the power spectrum of a standard MR image is mainly distributed along particular radial k-space directions. These directions often terminate before the k-space border has been reached. Some of them do not start from the k-space centre and extend to the k-space border. In order to take into account these opposite situations, a set of preliminary circular trajectories are collected. The circular trajectories allows the interception of the most important projections. By analyzing the collected data, it is possible to establish the desired set of projections before the image acquisition starts. For this reason, the acquisition process consists of the preliminarily collection of two concentric circular trajectories having 
Giuseppe Placidi; Recent Advances in Acquisition/Reconstruction Algorithms for Undersampled Magnetic Resonance Imaging, Journal of Biomedical Engineering and Medical Imaging, Volume 1, No 5, Oct (2014) , pp 53-70

the centre in the image k-space centre. The directions of the most informative projections can then be set by using the information acquired from the power spectra of these paths of coefficients. In particular, from the logarithm (used to reduce differences in scale) of the power spectra of the collected trajectories, the mean values are calculated and maxima above the mean value, in both curves, are used to indicate the presence of the most informative radial directions. The set of these angular directions is considered to be the optimal, most informative, set of projections to be collected in a standard way. Thought they require some preliminary time to collect the necessary information about the optimal angle set before the acquisition of radial projections started and, in some case, specialized hardware, the adaptive algorithms allow both the improvement in image quality and the reduction of the number of k-space coefficients with respect to other, non-adaptive, methods. Near optimal acquisition parameters are priory studied and set by using a numerical MRI simulation algorithm [85]. The adaptive methods proposed in [80-84] use a restoration/reconstruction method based on FFT and nearest neighbor interpolation [86]. Nearest neighbor interpolation is justified by the fact that close measured projections are very similar because of the used adaptive acquisition methods.

In principle, both sampling/reconstruction strategies, CS and the adaptive, appear reasonably, but it seems that they have to be considered separately. The question arising in the last period is to verify the possibility of using the characteristics of CS in an adaptive way. The advantage would be twofold. Classical CS, being a completely random sampling strategy that not uses information about the sample shape, needs that a minimum, fixed, number of samples is collected in order to avoid undersampling artifacts: the use of information about the sample shape furnished by an adaptive acquisition technique could reduce the number of necessary samples and to reach a near optimal, image dependent, value. On the other side, by verifying that for the adaptively collected samples the previously reported CS requirements (constraints) are satisfied, a CS non-linear reconstruction procedure could be used on these samples in order to obtain otimal image reconstruction. The possibility of using an hybrid, adaptive-CS, sampling strategy is, for the first time, proposed in [87], where it has been verified that the adaptively, radially collected, samples verify the CS constraints and a L1-norm based non-linear reconstruction can be used to obtain very accurate image reconstruction. After that, in [88,89], an alternative adaptive-CS method is presented that combines random sampling of Cartesian trajectories with an adaptive 2D acquisition of radial projections. It is based on the evaluation of the information content of a small percentage of the k-space data collected randomly to identify radial blades (similar to those used in PROPELLER $[39,40])$ of k-space coefficients having maxi-mum information content. The information content of each direction is evaluated by calculating an entropy function defined on the power spectrum of the projections. Besides that, the images are obtained by using a non linear reconstruction strategy, based on the homotopic L0-norm [75], on the sparse data. The method overcomes classical weighted CS in image quality, though a lower number of collected samples is used. The use of homotopic LO-norm minimization makes possible to obtain better reconstructions than by simple L1-norm. The same method is also applied on cardiac MRI data in [90] and very impressive results are obtained. Though the adaptive-CS method is capable to reduce the number of necessary samples below that required by classical CS, this number is still far to be optimal and can be further reduced. Work is currently in progress in order to verify how to reduce this number and how to apply the adaptive CS also to other sampling strategies (for example, pure cartesian, or spiral). 


\section{Conclusions}

Recently MRI has developed considerably into the directions of dynamic imaging and fMRI opening up several new fields of application, in particular referring to real-time imaging. Although these developments are generally promising their application can be limited by the compromise between temporal and spatial resolution. In order to improve temporal resolution, many sampling approaches use undersampling.

A review of the most effective methods for sparse sampling acquisition and reconstruction, with the aim of reducing the undersampling artifacts, have been presented. In particular, methods have been classified in two classes: those acquisition methods which do not use any restoration algorithm and those methods for which a restoration algorithm is essential.

A first consideration which can be made is that methods allowing to the second class are more complicated than those allowing to the first class but, in general, they perform better. Further considerations regard the last class of methods. In fact, it has been divided in two sub-classes: those reconstruction/restoration methods that reduce artifacts independently of the sample shape, and those acquisition/reconstruction/restoration methods that adapt their action by modifying the trajectories during the acquisition, i.e. the chosen trajectories (both in number and directions) are dependent on the sample shape. To the first subclass of methods allows also Compressed Sensing, a very breakthrough technique which, for some extends, appears as counterintuitive: images can be almost exactly reconstructed by a sparse set of its Fourier coefficients, below the Nyquist requirements, if they are compressible and the collected coefficients are casually collected in the k-space plane. The second subclass contains some adaptive methods: they allow a strong reduction of the collected data if the kspace paths are collected through the most informative directions (coefficients with maximum energy). Both CS and Adaptive methods require the images to be compressible, but they are completely different regarding the acquisition directions: in the first data are collected casually, in the second data are collected along the "most informative" directions, where energy is maximum. Hybrid methods, adaptive-CS strategies, are currently under investigation in order to obtain near optimal image reconstruction (as ensured by CS) with a reduced number of collected samples (as ensured by an adaptive sampling strategy).

\section{REFERENCES}

[1]. B.R.Rosen, J.W.Belliveau, D.Chien, "Perfusion imaging by nuclear magnetic resonance". Magn. Reson. Q. 5:263-281; (1989).

[2]. N.Wilke, C.Simm, J.Zhang, J.Ellermann, X.Ya, H.Merkle, G.Path, H.Ludemann, R.J.Bache, K.Ugurbil, "Contrast-enhanced first pass myocardial perfusion imaging: Correlation between myocardial blood flow in dogs at rest and during hyperemia", Magn. Reson. Med. 29:485-497; (1993).

[3]. V.M.Runge, J.F.Timoney, N.M.Williams, "Magnetic resonance imaging of experimental pyelonephritis in rabbits", Invest. Radiol., 32,696-704; (1997). 
Giuseppe Placidi; Recent Advances in Acquisition/Reconstruction Algorithms for Undersampled Magnetic Resonance Imaging, Journal of Biomedical Engineering and Medical Imaging, Volume 1, No 5, Oct (2014) , pp 53-70

[4]. A.H.Wilman, S.J.Riederer, B.F.King, J.P.Debbins, P.J.Rossman, R.L.Ehman, "Fluoroscopically triggered contrast-enhanced three-dimensional MR angiography with elliptical centric view order: Application to the renal arteries". Radiol. 205:137-146; (1997).

[5]. K.K.Kwong, J.W.Belliveau, D.A.Chesler, I.E.Goldberg, R.M.Weisskoff, B.P.Poncelet, D.N. Kennedy, B.E.Hoppel, M.S.Cohen, R.Turner, "Dynamic magnetic resonance imaging of human brain activity during primary sensory stimulation". Proc. Natl. Acad. Sci. USA. 89:5675-5679; (1992).

[6]. R.Lufkin, L.Teresi, L.Chiu, W.Hanafee, "A technique for MR guided needle placement". Am. J. Roentgenol. 151:193-196; (1988).

[7]. D.A.Leung, J.F.Debatin, S.Wildermuth, N.Heske, C.L.Dumoulin, "Real-time biplanar tracking for interventional MR imaging procedures". Radiology 197:485-488; (1995).

[8]. G.Adam, J.M.Neuerburg, A.Bucker, A.Glowinski, D.Vorwerk, a.Stargardt, J.Jvan Vaals, R.W.Gunther, "Interventional MR: First clinical experiments on a 1.5T system combined with C-arm fluoroscopy". Invest. Radiol. 32:191-197; (1997).

[9]. F.A.Jolesz, A.R.Bleier, P.Jakab, P.W.Ruenzel, K.Huttl, G.J.Jako, “MR imaging of laser tissue interaction". Radiology 168:249-253; (1988).

[10]. R.Matsumoto, A.M.Selig, V.M.Colucci, F.A.Jolesz, "MR monitoring during cryotherapy in the liver: Predictability of histologic outcome". J. Magn. Reson. Imag. 3:770-776; (1993).

[11]. J.Kettenbach, S.G.Silverman, K.Kuroda, Y.Nakajima, G.P.Zientara, P.Saiviroonporn, N. Hata, P.R.Morrison, S.G.Hushek, D.Gering, P.McL.Black, R.Kikinis, F.A.Jolesz, "Real-time monitoring and quantitative analysis of MR-guided laser ablations". In: Book of abstracts: Fifth Annual Scientific Meeting and Exhibition. ISMRM; 523, (1997).

[12]. C.L.Dumoulin, S.P.Souza, R.D.Darrow, "Real-time positioning of invasive devices using magnetic resonance". Magn. Reson. Med. 29:411- 415; (1993).

[13]. A.Glowinski, G.Adam, A.Bucker, J.M.Neuerburg, J.J.van Vaals, R.W.Gunther, "Catheter visualization using locally induced actively controlled field inhomogeneities". Magn. Reson. Med. 38:253-258; (1997).

[14]. G.Placidi, D.Franchi, L.Marsili, P.Gallo, "Development of an auxiliary system for the execution of vascular catheter interventions with a reduced radiological risk; system description and first experimental results", Comp. Meth. Progr. Biomed. 88 (2), 144-151, (2007).

[15]. G.Placidi, D.Franchi, A.Maurizi, A.Sotgiu, "Review on Patents about Magnetic Localisation Systems for in vivo Catheterizations", Recent Patents on Biomedical Engineering 2 (1), 58-64, (2009).

[16]. A.B.Kerr, J.M.Pauly, B.S.Hu, K.C.Li, C.J.Hardy, C.H.Meyer, A.Macovski, D.G.Nishimura, "Real-time interactive MRI on a conventional scanner". Magn. Reson. Med. 38:355-367; (1997).

[17]. V.Rasche, D.Holz, R.Proksa, "MR Fluoroscopy Using Projection Reconstruction Multi-Gradient-Echo (prMGE) MRI". Magn. Reson. Med. 42:324-334; (1999) 
[18]. J.H.Lee, B.A.Hargreaves, B.S.Hu, D.G.Nishimura, "Fast 3D imaging using variable-density spiral trajectories with applications to limb perfusion". Magn Reson Med; 50:1276-1285, (2003).

[19]. G.J.Marseille, R.de Beer, M.Fuderer, A.F.Mehlkopf, D.van Ormondt, "Nonuniform phase-encode distributions for MRI scan time reduction". J. Magn Reson B; 111:70-75, (1996).

[20]. J.Spiniak, A.Guesalaga, R.Mir, M.Guarini, P.Irarrazaval, "Undersampling k-space using fast progressive 3D trajectories". Magn Reson Med; 54:886-892, (2005).

[21]. K.Scheffler, J.Hennig, "Reduced circular field-of-view imaging", Magn Reson Med; 40:474-480, (1998).

[22]. J.R.Liao, J.M.Pauly, T.J.Brosnan, N.J.Pelc, "Reduction of motion artifacts in cine MRI using variabledensity spiral trajectories". Magn Reson Med; 37:569-575, (1997).

[23]. D.C.Peters, F.R.Korosec, T.M.Grist, W.F.Block, J.E.Holden, K.KVigen, C.A.Mistretta, "Undersampled projection reconstruction applied to MR angiography". Magn Reson Med; 43:91-101, (2000).

[24]. D.M.Spielman, J.M.Pauly, C.H.Meyer. "Magnetic resonance fluoroscopy using spirals with variable sampling densities". Magn Reson Med;34:388-394, (1995).

[25]. J.H.Gao, J.Xiong, S.Lai, E.M.Haacke, M.G.Woldorff, J.Li, P.T.Fox. "Improving the temporal resolution of functional MR imaging using keyhole techniques”. Magn Reson Med; 35:854-860 (1996).

[26]. K.K.Vigen, D.C.Peters, T.M.Grist, W.F.Block, C.A.Mistretta "Undersampled projection-reconstruction imaging for time-resolved contrast-enhanced imaging". Magn Reson Med; 43:170-176, (2000).

[27]. A.V.Barger, W.F.Block, Y.Toropov, T.M.Grist, C.A.Mistretta, "Time resolved contrast-enhanced imaging with isotropic resolution and broad coverage using an undersampled 3D projection trajectory". Magn Reson Med; 48:297-305, (2002).

[28]. K.P.Pruessmann, M.Weiger, M.B.Scheidegger, P.Boesiger. "SENSE: sensitivity encoding for fast MRI". Magn Reson Med;42:952-962, (1999).

[29]. D.K.Sodickson, W.J.Manning, "Simultaneous acquisition of spatial harmonics (SMASH): fast imaging with radiofrequency coil arrays". Magn Reson Med;38:591-603, (1997).

[30]. M.A.Griswold, P.M.Jakob, R.M.Heidemann, M.Nittka, V.Jellus, J.Wang, B.Kiefer, A.Haase, "Generalized autocalibrating partially parallel acquisitions (GRAPPA)," Magn. Reson. Med., 47, 1202-1210, (2002).

[31]. D.R.Thedens, P.Irarrazaval, T.S.Sachs, C.H.Meyer, D.G.Nishimura, "Fast magnetic resonance coronary angiography with a three-dimensional stack of spirals trajectory". Magn Reson Med;41:1170-1179, (1999).

[32]. C.M.Tsai, D.G.Nishimura, "Reduced aliasing artifacts using variable density k-space sampling trajectories", Magn Reson Med;43:452-458, (2000).

[33]. P.Irarrazaval, J.M.Santos, M.Guarini, D.Nishimura, "Flow properties of fast three-dimensional sequences for MR angiography". Magn Reson Imag.;17:1469-1479 (1999) 
Giuseppe Placidi; Recent Advances in Acquisition/Reconstruction Algorithms for Undersampled Magnetic Resonance Imaging, Journal of Biomedical Engineering and Medical Imaging, Volume 1, No 5, Oct (2014) , pp 53-70

[34]. S.T.Wong, M.S.Roos, "A strategy for sampling on a sphere applied to 3D selective RF pulse design", Magn Reson Med;32:778-784, (1994).

[35]. E.B.Welch, A.Manduca, R.C.Grimm, H.A.Ward, Jr.C.R. Jack, "Spherical navigator echoes for full 3D rigid body motion measurement in MRI", Magn Reson Med;47:32-41 (2002).

[36]. Y.Shu, A.M.Elliott, S.J.Riederer, M.A.Bernstein, "3D RINGLET: spherical shells trajectory for selfnavigated 3D MRI". In: Proceedings of the 13th Annual Meeting of ISMRM, Miami Beach, FL, USA, 2693, (2005).

[37]. Y.Shu, S.J.Riederer, M.A.Bernstein, "Motion correction properties of the shells k-space trajectory". Magn Reson Imag., 24:739-749, 2006.

[38]. Y.Shu, S.J.Riederer, M.A.Bernstein, "Three-Dimensional MRI with an Undersampled Spherical Shells Trajectory", Magn. Reson. Med. 56:553-562 (2006)

[39]. J.G.Pipe, "Motion correction with PROPELLER MRI: Application to head motion and free-breathing cardiac imaging". Magn Reson Med, 42, 963-969 (1999)

[40]. J.G.Pipe, V.G.Farthing, K.P.Forbes, "Multishot diffusion-weighted FSE using PROPELLER MRI". Magn Reson Med, 47, 42-52 (2002).

[41]. K.Arfanakis, A.A.Tamhane, J.G.Pipe, M.A.Anastasio, "k-Space Undersampling in PROPELLER Imaging", Magn. Reson. Med., 53, 675-683 (2005)

[42]. J.J.van Vaals, M.E.Brummer, W.T.Dixon, H.H.Tuithof, H.Engels, R.C.Nelson, B.M.Gerety, J.L.Chezmar, J.A.den Boer, "Keyhole method for accelerating imaging of contrast agent uptake," J. Magn. Reson. Imag., 3, 671-675, (1993).

[43]. R.A.Jones, O.Haraldseth, T.B.Muller, P.A.Rinck, A.N.Oksendal, "K-space substitution: A novel dynamic imaging technique," Magn. Reson. Med., 29, 830-834, (1993).

[44]. S.Di Giuseppe, G.Placidi, J.Brivati, M.Alecci, A.Sotgiu, "Pulsed EPR imaging: image reconstruction using selective acquisition sequences", Phys. Med. Biol., 44, N137-N144, (1999).

[45]. D.Franchi, A.Sotgiu, G.Placidi, "A novel acquisition-reconstruction algorithm for surface magnetic resonance imaging", Magn. Reson. Imag, 26 (9), 1303-1309, (2008).

[46]. A.G.Webb, Z.P.Liang, R.L.Magin, P.C.Lauterbur, "Applications of reduced-encoding MR imaging with generalized-series reconstruction (RIGR)," J. Magn. Reson. Imag., 3, 925-928, (1993).

[47]. J.Tintera, G.Schaub, J.Gawehn, P.Stoeter, "Functional MRI with keyhole technique". Human Brain Mapping s1: 124; (1995).

[48]. J.H.Gao, J.Xiong,; S.Lai,; E.M.Haacke, M.G.Woldorff, "Improving the temporal resolution of functional MR imaging using keyhole techniques". Magn. Reson. Med. 35:854-860; (1996).

[49]. J.Xiong, P.T.Fox, J.H.Gao, "The effects of k-space data undersampling and discontinuities in keyhole functional MRI”. Magn. Reson. Imag., 17, 109-119, (1999) 
[50]. J.J.van Vaals, G.H.van Yperen,; R.W.de Boer, "Real-time MR imaging using the LoLo (Local Look) method for interactive and interventional MR at 0.5T and 1.5T". In: Book of abstracts: Second Annual Meeting of the Society of Magnetic Resonance Imaging. SMR; 421, (1994).

[51]. X.Hu, T.Parrish, "Reduction of field of view imaging". Magn. Reson. Med. 31:691-694; (1994).

[52]. M.E.Brummer, D.Moratal-Perez, C.Y.Hong, R.I.Pettigrew, J.Millet-Roig, W.T.Dixon, “Noquist: Reduced field-of-view imaging by direct Fourier inversion," Magn. Reson. Med., 51, 331-342, (2004).

[53]. K.Scheffler, J. Hennig, "Reduced Circular Field-of-View Imaging." In: Book of abstracts: Sixth Annual Scientific Meeting and Exhibition. ISMRM; 180, 1998.

[54]. S.Weiß, V.Rasche, "Projection-Reconstruction Reduces FOV Imaging", Magn. Reson. Imag., 17, 517525, (1999)

[55]. D.C.Peters, M.A.Guttman, A.J.Dick, V.K.Raman, R.J.Lederman, E.R.McVeigh, "Reduced Field of View and Undersampled PR Combined for Interventional Imaging of a Fully Dynamic Field of View", Magn. Reson. Med., 51, 761-767 (2004).

[56]. H.Sedarat, A.B.Kerr, J.M.Pauly, D.G.Nishimura, "Partial-FOV reconstruction in dynamic spiral imaging," Magn. Reson. Med., 43, 429-439, (2000).

[57]. B.Madore, G.H.Glover, N.J.Pelc, "Unaliasing by Fourier-encoding the overlaps using the temporal dimension (UNFOLD), applied to cardiac imaging and fMRI", Magn. Reson. Med., 42, 813-828, (1999).

[58]. J.Tsao, “On the UNFOLD method," Magn. Reson. Med., 47, 202-207, (2002).

[59]. J.Tsao, P.Boesiger, K.P.Pruessmann, "k-t BLAST and k-t SENSE: dynamic MRI with high frame rate exploiting spatiotemporal correlations," Magn. Reson. Med., 50, 1031-1042, (2003).

[60]. Q.S.Xiang R.M.Henkelman, "K-space description for MR imaging of dynamic objects," Magn. Reson. Med., 29, 422-428 (1993).

[61]. M.S.Hansen, C.Baltes, J.Tsao, S.Kozerke, K.P.Pruessmann, H.Eggers, "k-t BLAST reconstruction from non-Cartesian k-t space sampling," Magn. Reson. Med., 55, 85-91, (2006).

[62]. C.H.Meyer, B.S.Hu, D.G.Nishimura, A. Macovski, “Fast spiral coronary artery imaging," Magn. Reson. Med., 28, 202-213, (1992).

[63]. T.Shin, J.F.Nielsen, K.S.Nayak, ”Accelerating Dynamic Spiral MRI by Algebraic Reconstruction From Undersampled k-t Space", IEEE Trans. Med. Imag., 26, 917-924, (2007)

[64]. Y.Hu, G.H.Glover, "Increasing Spatial Coverage for High-Resolution Functional MRI", Magn Reson Med, 61:716-722 (2009)

[65]. Y. Hu, G.H.Glover, "Three-dimensional spiral technique for high-resolution functional MRI", Magn Reson Med;58:947-951, (2007). 
Giuseppe Placidi; Recent Advances in Acquisition/Reconstruction Algorithms for Undersampled Magnetic Resonance Imaging, Journal of Biomedical Engineering and Medical Imaging, Volume 1, No 5, Oct (2014) , pp 53-70

[66]. G.Placidi, A.Sotgiu, "A novel restoration algorithm for reduction of undersampling artifacts from Magnetic Resonance Images", Magn. Reson. Imag., 22, 1279-1287, (2004).

[67]. G.Placidi, M.Alecci, S.Colacicchi, A.Sotgiu, "Fourier reconstruction as a valid alternative to filtered back projection in iterative applications: implementation of Fourier spectral spatial EPR imaging", J. Magn. Reson., 134, 280-286, (1998).

[68]. G.Placidi, "Constrained Reconstruction for Sparse Magnetic Resonance Imaging", Proc. WC2009, September 7-12, Munich, Germany (2009).

[69]. G.Placidi, M.Alecci, A.Sotgiu, "Post-processing noise removal algorithm for magnetic resonance imaging based on edge detection and wavelet analysis", Phys. Med. Biol., 48 (13), 1987-1995, (2003).

[70]. E.Candès, J.Romberg, T.Tao, "Robust uncertainty principles: Exact signal reconstruction from highly incomplete frequency information". IEEE Trans Inf Theory, 52, 489-509, (2006).

[71]. D.Donoho, "Compressed sensing". IEEE Trans Inf Theory, 52, 1289-1306, (2006)

[72]. E.Candès and T.Tao, "Near optimal signal recovery from random projections: Universal encoding strategies?" IEEE Trans. Inform. Theory, 52, pp.5406-5425, (2006).

[73]. M.Lustig, D.Donoho, J.M. Pauly, "Sparse MRI: The Application of Compressed Sensing for Rapid MR Imaging", Magn Reson Med, 58, 1182-1195 (2007)

[74]. M.Lustig, D.L.Donoho, J.M.Santos, J.M.Pauly, “Compressed Sensing MRI”, IEEE Sign. Proc. Magazine, 72-82, (2008)

[75]. J.Trzasko, A.Manduca, "Highly Undersampled Magnetic Resonance Image Reconstruction via Homotopic lo-Minimization", IEEE Trans. Med. Imag., 28, 106-121, (2009)

[76]. T.Knopp, S.Kunis, D.Potts, "A Note on the Iterative MRI Reconstruction from Nonuniform k-Space Data", Intern. J. Biomed. Imag., 2007, 1-9, (2007).

[77]. J.D.O'Sullivan, "A fast sinc function gridding algorithm for Fourier inversion in computer tomography," IEEE Trans. Med. Imag., 4, 200-207, (1985).

[78]. K.T.Block, M.Uecker, J.Frah, "Undersampled Radial MRI with Multiple Coils. Iterative Image Reconstruction Using a Total Variation Constraint", Magn Reson Med, 57, 1086-1098, (2007)

[79]. I.Contreras, A.Guesalga, M.P.Fernandez, M.Guarini, P.Irarrazaval, MRI fast tree log scanning with helical undersampled projection acquisitions, Magn. Reson. Imag., 20, 781-787, (2002)

[80]. G.Placidi, M.Alecci, A.Sotgiu, "Theory of Adaptive Acquisition Method for Image Reconstruction from Projections and Application to EPR Imaging", J. Magn. Reson., Series B, 108, 50-57, (1995).

[81]. G.Placidi, M.Alecci, A.Sotgiu, "Metodo perfezionato di acquisizione di dati nel dominio della frequenza spaziale per la ricostruzione di immagini bidimensionali, in particolare di risonanza magnetica nucleare, e relativo apparato", Italian Patent No. RM98A000217, Issued April 3, (1998). 
[82]. G.Placidi, M.Alecci, and A.Sotgiu, " $\omega$-Space adaptive Acquisition Technique for Magnetic Resonance Imaging from Projections", J. Magn. Reson., 143, 197-207, (2000).

[83]. G.Placidi, "Adaptive compression algorithm from projections: Application on medical greyscale images", Comp. Biol. Med., 39 (11), 993-999, (2009).

[84]. G.Placidi "Circular Acquisition to Define the Minimum Set of Projections for Optimal MRI Reconstruction" Lecture Notes in Computer Science, 6026, 254-262 (2010).

[85]. G Placidi, M Alecci, A Sotgiu, "A general Algorithm for Magnetic Resonance Imaging Simulation: a Versatile Tool to Collect Information about Imaging Artefacts and New Acquisition Techniques", Studies in health technology and informatics, 13-17, (2002).

[86]. G.Placidi, M.Alecci, A.Sotgiu, "Angular Space-Domain Interpolation for Filtered Back Projection Applied to Regular and Adaptively Measured Projections". J. Magn. Reson., Series B, (110), 75-79, (1996).

[87]. G.Placidi, “MRI: Essentials for Innovative Technologies”, CRC Press, (2012).

[88]. L.Ciancarella, D.Avola, E.Marcucci, G.Placidi, A hybrid sampling strategy for Sparse Magnetic Resonance Imaging, Di Giamberardino et al. Editors, CRC Press, 285-289, (2012).

[89]. L.Ciancarella, D.Avola, G.Placidi, "Adaptive Sampling and Reconstruction for Sparse Magnetic Resonance Imaging", Computational Modeling of Objects Presented in Images, Lecture Notes in Computational Vision and Biomechanics, Springer, 15, 115-130, (2014).

[90]. G.Placidi, D.Avola, L.Cinque, G.Macchiarelli, A.Petracca, M.Spezialetti, "Adaptive Sampling and Non Linear Reconstruction for Cardiac Magnetic Resonance Imaging", Computational Modeling of Objects Presented in Images. Fundamentals, Methods, and Applications, Lecture Notes in Computer Science, 8641, 24-35, (2014). 